\title{
The Clinical and Computed Tomography Findings of Patients with COVID-19
}

\author{
Afsin Ipekci ${ }^{1, *}$, Yonca Senem Akdeniz ${ }^{1}$, Onur Tutar ${ }^{2}$, Sabri \\ Sirolu' ${ }^{2}$, Osman Simsek ${ }^{3}$, Seda Özkan ${ }^{1}$
}

\author{
${ }^{1}$ Department of Emergency Medicine, \\ Cerrahpasa Faculty of Medicine, Istanbul \\ University-Cerrahpasa, Istanbul, Turkey \\ ${ }^{2}$ Department of Radiology, Cerrahpasa \\ Faculty of Medicine, Istanbul \\ University-Cerrahpasa, Istanbul, Turkey \\ ${ }^{3}$ Department of General Surgery, \\ Cerrahpasa Faculty of Medicine, Istanbul \\ University-Cerrahpasa, Istanbul, Turkey

\section{*Correspondence} \\ afsin.ipekci@istanbul.edu.tr \\ (Afsin Ipekci)
}

\begin{abstract}
Objective: To evaluate the demographic, clinical data and, computed tomography findings of patients diagnosed COVID-19. Methods: Patients who had COVID 19 suspicion in the emergency department of the university hospital in Istanbul, Turkey, between March 20, 2020, and April 1, 2020, were scanned. Demographic, characteristics, and computed tomography findings of patients with positive RT-PCR test results were analyzed. Results: The mean age of patients was 51.27 (6.45) years, and $72.5 \%$ were male. The median admission period of patients was $4(1-10)$ days, and the mean length of hospital stay was 10.49 (6.6) days. The mean CT result time was 33.24 (11.56) minutes, and RT-PCR was 35.53 (14.36) hours. The most common complaint was a fever. Furthermore, shortness of breath and dry cough was other evident complaints. Only $7.8 \%$ of patients were asymptomatic. In $84.3 \%$ and $80.5 \%$ of patients had increased C-reactive protein levels and increased ferritin levels, while in $41.2 \%$ of patients had decreased lymphocyte count. Bilateral lung involvement, multifocal localized lung lesions, peripheral and central distribution of lesions were detected in most patients. Lesions were located at the posterior lung in more than half of the patients. The rate of involvement of the lower lobes was higher. Some $84.5 \%$ of the patients had two or more lobe involvements. Ground glass density (94.1\%), consolidation (80.4\%), pleural thickening (64.7\%), crazy paving pattern (52.9\%), vascular enlargement $(47.1 \%)$, halo sign $(43.1 \%)$, and air bronchogram (33.3\%) were the most seen lesions. Conclusion: Computed tomography could be helpful in coordination with the clinical and laboratory parameters for early decision and isolation of patients with suspected COVID19 until RT-PCR test results obtained.
\end{abstract}

\section{Keywords}

COVID-19, SARS-CoV-2, Coronavirus, Computed tomography

\section{Introduction}

Coronavirus disease 2019 (COVID-19), which is a severe acute respiratory disease caused by coronavirus 2 (SARSCoV-2), first appeared in Wuhan city of Hubei province of China in December 2019 and spread rapidly to other countries [1]. The World Health Organization (WHO) declared the disease as an emergency public health problem on January 30, 2020, after that, was announced as pandemic on March 11, 2020 [2]. According to data of an online virus tracker created by The Lancet and organized by Johns Hopkins University, 5,370,893 confirmed COVID19 cases, and 343,617 deaths have been reported worldwide in 188 countries [3]. According to the data of the Republic of Turkey Ministry of Health, 156.827 confirmed cases, and 4.340 deaths are reported to date [4].

A real-time reverse transcription-polymerase chain reaction (RT-PCR) of the virus's nucleic acid is accepted as the standard test in the diagnosis of COVID-19 disease, hospitalization, or isolation decision. Studies reported a 
TA B L E 1. Demographic, clinical data, and laboratory results of the patients.

\begin{tabular}{|c|c|}
\hline & Patients $(n=51)$ \\
\hline Mean age, years (SD) & $51.27(14.65)$ \\
\hline Men & $37(72.5 \%)$ \\
\hline Women & $14(27.5 \%)$ \\
\hline Median admission period, days (range) & $4(1-10)$ \\
\hline Mean Lenght of the hospital stay, days (SD) & $10.49(6.6)$ \\
\hline Mean CT result time, minutes (SD) & $33.24(11.56)$ \\
\hline \multirow[t]{2}{*}{ Mean RT - PCR result time, hours (SD) } & $35.53(14.36)$ \\
\hline & Sign and symptoms \\
\hline Fever & $30(58.8 \%)$ \\
\hline Dry cough & $21(41.2 \%)$ \\
\hline Dispnea & $24(47.1 \%)$ \\
\hline Fatigue, weakness & $16(31.4 \%)$ \\
\hline Chest or back pain & $6(11.8 \%)$ \\
\hline Diarrhea & $5(9.8 \%)$ \\
\hline \multirow[t]{2}{*}{ Without any symptoms } & $4(7.8 \%)$ \\
\hline & Vital signs \\
\hline Fever, ${ }^{\circ} \mathrm{C}$, mean $(\mathrm{SD})$ & $37.68(0.95)$ \\
\hline Heart rate, beats per minute & $86(50-115)$ \\
\hline Respiratory rate per minute & $20(16-40)$ \\
\hline Systolic blood pressure, $\mathrm{mmHg}$ & $115(90-150)$ \\
\hline Diastolic blood pressure, $\mathrm{mmHg}$ & $70(60-90$ \\
\hline \multirow[t]{2}{*}{$\mathrm{SPO}_{2}, \%$} & $96(82-100)$ \\
\hline & Laboratory tests \\
\hline Median leucocyst count, (range) $(\times 103 / \mu \mathrm{L}$, normal range $4.3-10.3)$ & $5.8(1.1-25)$ \\
\hline Increased & $4(7.8 \%)$ \\
\hline Normal & $44(86.3 \%)$ \\
\hline Decreased & $3(5.9 \%)$ \\
\hline Median lymphocyte count (range) $\left(\times 10^{3} / \mu \mathrm{L}\right.$, normal range $\left.1.3-3.5\right)$ & $1.4(0.5-3)$ \\
\hline Decreased & $21(41.2 \%)$ \\
\hline Normal & $30(58.8 \%)$ \\
\hline Median $\mathrm{C}$ - reactive protein level (range) $(\mathrm{mg} / \mathrm{L}$, normal range $<5$ ) & $30.5(0.47-396)$ \\
\hline Increased & $43(84.3 \%)$ \\
\hline Normal & $8(15.7 \%)$ \\
\hline Median ferritin level (range) (ng/ml, normal range $15-150)$ & $431(48.5-2296)$ \\
\hline Increased & $33(80.5 \%)$ \\
\hline Normal & $8(19.5 \%)$ \\
\hline Median D - Dimer level (range) (mg/L, normal range 0 - 0.5$)$ & $0.56(0.20-80)$ \\
\hline Increased & $30(65.2 \%)$ \\
\hline \multirow[t]{2}{*}{ Normal } & $16(34.8 \%)$ \\
\hline & Decision \\
\hline Outpatients follow-up & $5(9.8 \%)$ \\
\hline Hospilatized in general ward & $38(74.5 \%)$ \\
\hline Hospilatized in the intensive care unit & $8(15.7 \%)$ \\
\hline \multicolumn{2}{|l|}{ Outcome } \\
\hline Survived & $47(92.2 \%)$ \\
\hline Non - survived & $4(7.8 \%)$ \\
\hline
\end{tabular}

high false-negative result rate and low sensitivity of the RT-PCR test. This resulted in increased necessity and use of non-contrasted chest computed tomography (CT), later having higher sensitivity, faster results procurement, and more practical in usage [5-7].

We aimed to evaluate the demographic, clinical data, and 
TA B L E 2. Features of chest CT findings of patients according to the admission period.

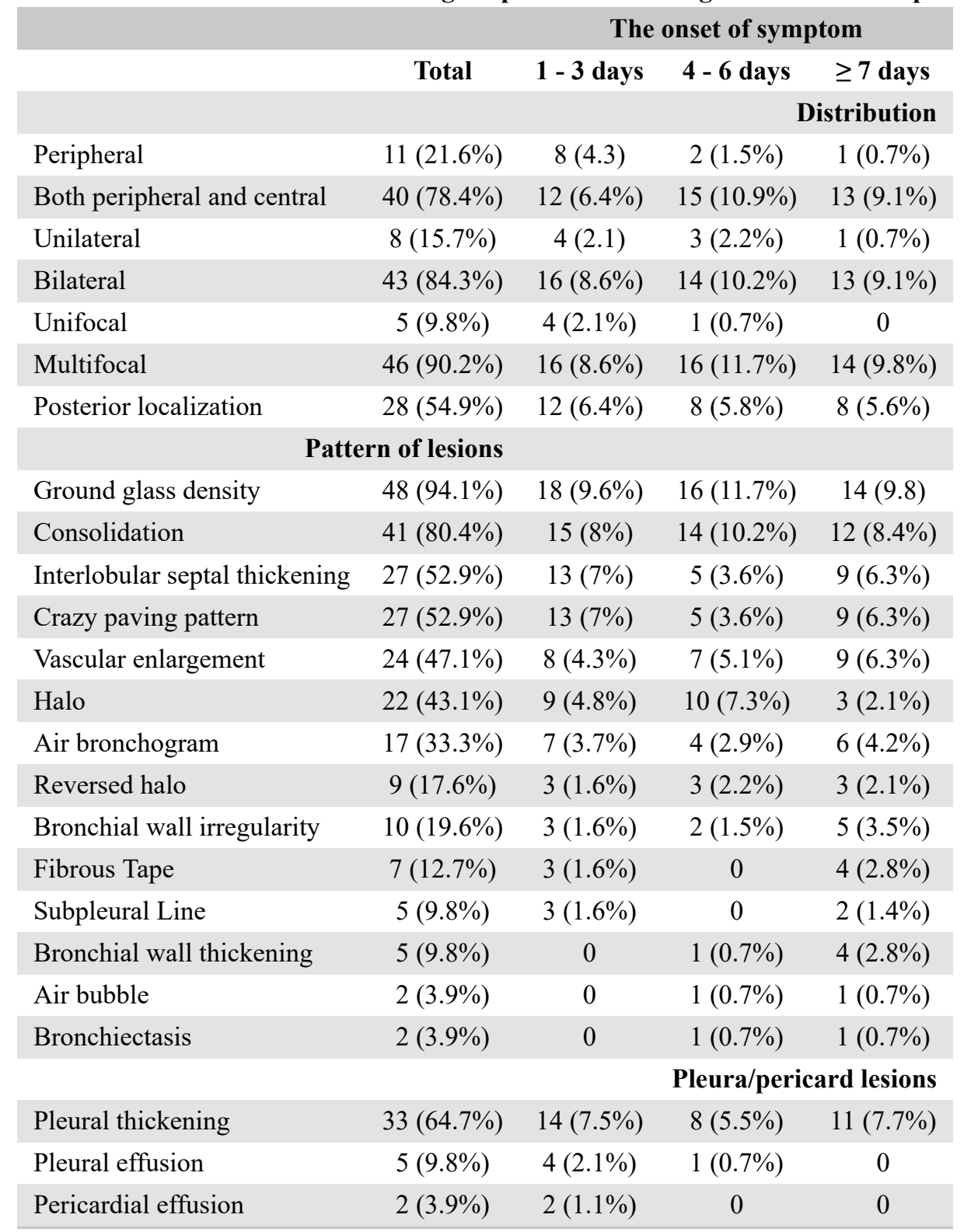

CT findings of the patients who had positive RT-PCR test results.

\section{Materials and methods}

This descriptive study was performed retrospectively scanning of 87 patient's files who were older than 18 years of age and having COVID 19 suspicion in the emergency department of the university hospital in Istanbul, Turkey, between March 20, 2020, and April 1, 2020.

Research ethics committee approval was obtained from the Istanbul University-Cerrahpasa ethical committee with a number of 58635 .

Patients with positive RT-PCR test results were included in the study. Age, gender, complaints, the onset of symptoms, vital signs, a decision about follow-up, length of the hospital stay, laboratory results and, the time interval of obtaining RT-PCR and CT results were recorded and analyzed. Patients were divided into three, 1 - 3 days, 4
- 6 days, and seven or more days, according to the onset of symptoms, and chest CT findings were recorded.

\subsection{CT image acquisition and interpretation}

CT images of patients were taken on a spine position by using a 128 detector CT device (Somatom Definition CT, Siemens Healthcare, Forcheim, Germany) and lobe of lesion distribution, with a minimum slice thickness of $1 \mathrm{~mm}$. CT findings of patients were interpreted by two radiologists with 5 and 15 years of experience.

CT findings focused on the lesion features of each patient, included (a) distribution characteristics, (b) lobe of lesion distribution, (c) a number of lobes involved (d) patterns of the lesion (e.g., ground glass opacification, crazy paving pattern, consolidation, halo sign, interlobular septal thickening, air bronchogram), and (e) pleural/pericardial findings (e.g., adjacent pleura thickening, pleural effusion, 
TA B L E 3. Location and the number of the involved lobes.

\begin{tabular}{|c|c|c|c|c|}
\hline & \multirow[b]{2}{*}{ Total } & \multicolumn{3}{|c|}{ The onset of symptom } \\
\hline & & 1 - 3 days & 4 - 6 days & $\geq 7$ days \\
\hline \multicolumn{5}{|c|}{ Affected lobes } \\
\hline Right upper lobe & $38(74.5 \%)$ & $15(19.7 \%)$ & $11(18.3 \%)$ & $12(18.5 \%)$ \\
\hline Right middle lobe & $37(72.5 \%)$ & $14(18.4 \%)$ & $10(16.7 \%)$ & $13(20 \%)$ \\
\hline Right lower lobe & $46(90.2 \%)$ & $17(22.4 \%)$ & $15(25.0 \%)$ & $14(21.5 \%)$ \\
\hline Left upper lobe & $37(72.5 \%)$ & $14(18.4 \%)$ & $10(16.7 \%)$ & $13(20 \%)$ \\
\hline Left lower lobe & $43(84.3 \%)$ & $16(21.1 \%)$ & $14(23.3 \%)$ & $13(20 \%)$ \\
\hline \multicolumn{5}{|c|}{ Number of the involved lobe } \\
\hline \multicolumn{2}{|c|}{ Median number of involved lobe } & & & $5(1-5)$ \\
\hline 1 lobe & $8(15.7 \%)$ & $5(25 \%)$ & $2(11.8 \%)$ & $1(7.1 \%)$ \\
\hline 2 lobes & $4(7.8 \%)$ & $1(5.0 \%)$ & $3(17.6 \%)$ & 0 \\
\hline 3 lobes & $1(2.0 \%)$ & 0 & $2(5.9 \%)$ & 0 \\
\hline 4 lobes & $12(23.5 \%)$ & $5(25 \%)$ & $6(35.3 \%)$ & $1(7.1 \%)$ \\
\hline 5 lobes & $26(51.0 \%)$ & $9(45.0 \%)$ & $5(29.4 \%)$ & $12(85.7 \%)$ \\
\hline
\end{tabular}

pericardial effusion).

\subsection{Statistical analysis}

Statistical analysis of data was performed on SPSS 21.0 for the Windows program. Kolmogorov-Smirnov test was used to assess the normality of distribution. Normally distributed data were presented as mean (SD), non-normally distributed data as median (IQR), and categorical variables as frequency $(\%)$.

\section{Results}

A total of 51 patients with positive RT-PCT test results were included in the study. The mean age of the patients was $51.27(6.45)$ years, and $72.5 \%$ were male. The median admission period of the patients was 4 (1-10) days, and the mean length of hospital stay was 10.49 (6.6) days. While the mean time interval of obtaining the CT result was 33.24 (11.56) minutes, the mean time interval of obtaining RTPCR results was 35.53 (14.36) hours. The most common complaint was fever observed in $58.8 \%$ of patients. Furthermore, shortness of breath and dry cough was evident in $47.1 \%$ and $41.2 \%$ of patients, respectively. Only $7.8 \%$ of patients were admitted without any symptoms. In $74.5 \%$ of the patients were hospitalized in the general ward, while $15.7 \%$ were hospitalized in the intensive care unit. In $84.3 \%, 80.5 \%$ and $41.2 \%$ of the patients had increased Creactive protein levels, increased ferritin levels, and decreased lymphocyte count, respectively (Table 1 ).

Bilateral lung involvement (84.3\%), multifocal localized lung lesions $(90.2 \%)$, peripheral and central distribution $(78.4 \%)$ of lesions were detected in the majority of patients. Lesions located peripheral, unilateral, and unifocal were seen more common in patients admitted in 1 - 3 days. Ground glass density $(94.1 \%)$, consolidation $(80.4 \%)$, and crazy paving pattern $(52.9 \%)$ were the most seen lesions.
Besides, vascular enlargement (47.1\%), halo (43.1\%), air bronchogram (33.3\%) and, accompanying pleural thickening $(64.7 \%)$ were other important lesions observed in patients (Table 2, Fig. 1).

Although the involvement of all lobes was detected, the involvement of the lower lobes was higher. In $84.5 \%$ of the patients, two or more lobe involvements were detected. Five lob involvements one lobe involvement were seen in $85.7 \%(\mathrm{n}=12)$ and $7.1 \%(\mathrm{n}=1)$ of patients admitted in 7 or more days (Table 3 ).

\section{Discussion}

Early diagnosis of COVID-19 is critical in controlling the course of the disease, reducing its infectiousness, and improving treatment strategy. Fever, dry cough, shortness of breath, myalgia, and weakness were the most common observed complaints, and acute respiratory distress syndrome was seen $17-29 \%$. Sore throat, rhinorrhea, chest pain, hemoptysis, conjunctival congestion, diarrhea, nausea, and vomiting were less common. Besides, the proportion of patients admitted without any symptoms was reported as $11 \%$ [8-11]. Fever was the most common complaint, but $7.8 \%$ of patients were asymptomatic in our study. Even if the majority of patients have some symptoms, the diagnosis of COVID-19 should be considered in patients with no symptoms for the isolation process to reduce the infectiousness of disease.

Lymphopenia (33 - 50\%), high C-reactive protein level (42 - 60\%), and high D-dimer level were reported as common and important laboratory parameters [9, 12-14]. Data obtained in our study were similar to the literature; however, high ferritin level was measured in $80.5 \%$ of patients.

The RT-PCR test results are affected by many external factors such as sampling location and time, personnel, and the performance of the kit studied. Also, the RT-PCR test's 


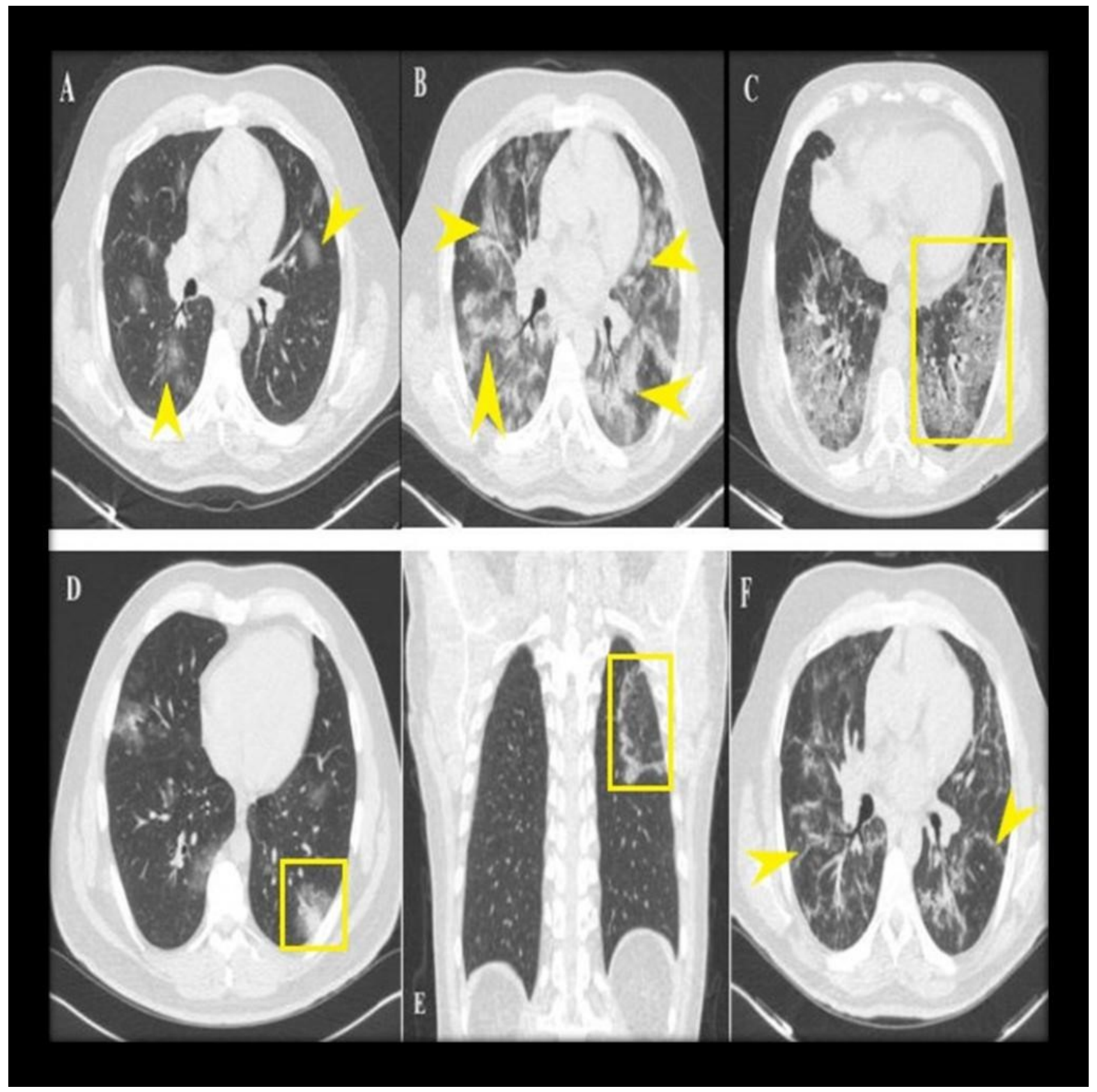

F I G U R E 1. Various lesions of included patients. Yellow arrowheads and boxes indicated the lesions. A: multiple patchy ground glass densities; B: large consolidation areas in the bilateral lung; C: crazy paving pattern; D: halo sign; E: reversed halo sign; F: fibrous tapes.

sensitivity was determined in the range of $30-60 \%$ in the studies [5, 15]. Even if we were not able to estimate the sensitivity of the RT-PCR test, the mean time interval of obtaining test results was 35.53 (14.36) hours in our study. Waiting for the RT-PCR test results to make a decision, a long time interval of obtaining test results might delay and prolong the hospitalization, isolation, and drug treatment, especially in patients without symptoms.

Chest CT is available in many centers. It is also easier to use, more practical and gives faster results than the RTPCR test. Also, the sensitivity of CT was determined in $97 \%$ and $70 \%$ patients with positive and negative RT-PCR test [7]. In addition, repetitive use of CT has been reported to be useful in evaluating the course of the disease and subsequent monitoring of the treatment [16]. Even if we were not able to detect negative CT findings in patients with positive RT-PCR test results, we found that the mean time interval of obtaining CT results was 33.24 (11.56) minutes. In our study, $90.1 \%$ and $9.9 \%$ of patients were hospitalized and isolated at home according to CT results before obtaining RT-PCR results. We gained approximately 1.5 days for drug treatment and isolation until obtaining RTPCR results.

Characteristic CT findings were found in the course of the disease. Especially the different degrees of ground glass density is reported to be the most prominent feature seen 
in patients with $85-98 \%$. Vascular enlargement in $71.3 \%$ cases, consolidation in $40-68 \%$ and, crazy paving pattern in $5-36 \%$, along with pleural thickening, were identified as other common lesions. In addition to these lesions, more rarely, halo sign, reverse halo sign, fibrous tape, air bubble, and lymphadenopathy have been reported [6, 9, 17-19]. In the distribution of lesions, bilateral lung involvement, peripheral location, multifocal distribution are the most common forms. Although the disease affects all lobes of the lung, the involvement of the lower lobes has been reported higher than other lobes. Also, the majority of patients had two or more lobe involvement [18-21]. In our study, $84.3 \%$ and $90.2 \%$ of patients had bilateral lung involvement and multifocal localized lesions. $84.5 \%$ of patients had two or more lobe involvement, similar to studies. Peripheral, unifocal and, unilateral localized lesions were common in patients admitted in 1 - 3 days, in contrast to patients admitted in 7 or more days.

There were some limitations in our study. The most important limitation of our study was that the RT-PCR positive patients without CT findings or the RT-PCR negative patients with CT findings were not included. Also, limited and short time period, less number of patients, and the absence of CT findings during the course of the disease were other limitations of our study.

\section{Conclusion}

Chest CT is easy to perform and gives faster results, which is a more practical and high sensitive manner. It is an important imaging method that could be helpful in coordination with the clinical and laboratory parameters in the diagnosis of COVID-19 disease. Moreover, CT might be useful for the isolation of patients in the centers and endemic regions where RT-PCR test results obtain late.

\section{ACKNOWLEDGEMENTS}

The authors received no financial support for the research, authorship, and/or publication of this article. This study was supported by the Department of Emergency Medicine, Radiology, and General Surgery of Cerrahpasa Faculty of Medicine, Istanbul University-Cerrahpasa, Istanbul, Turkey.

\section{CONFLICT OF INTEREST}

The authors declare no conflict of interest.

\section{REFERENCES}

[1] Zhu N, Zhang D, Wang W, et al. A novel coronavirus from patients with pneumonia in China, 2019. N Engl J Med. 2020;382:727-733.

[2] World Health Organization (2020) Coronavirus disease 2019 (COVID-19) situation report-39. World Health Organization,
Geneva. 2020. accessable on: https://www. who.int/docs/ default-source/coronaviruse/situation-reports/ 20200228-sitrep-39- covid-19.pdf? sfvrsn=5bbf3e7d 2 .

[3] John Hopkins University Coronavius Research Center. 2020. accessable on: https://coronavirus. jhu.edu/map.html.

[4] T.C Sağlık Bakanlığı Türkiye'de güncel durum. 2020. accessable on: https://covid19.saglik.gov.tr/.

[5] Xie X, Zhong Z, Zhao W, et al. Chest CT for typical 2019-nCoV pneumonia: relationship to negative RT-PCR testing. Radiology. 2020.

[6] Huang P, Liu T, Huang L, et al. Use of Chest CT in Combination with Negative RT-PCR Assay for the 2019 Novel Coronavirus but High Clinical Suspicion. Radiology. 2020.

[7] Fang Y, Zhang H, Xie J, et al. Sensitivity of Chest CT for COVID-19: Comparison to RT-PCR. Radiology. 2020.

[8] Ai T, Yang Z, Hou H, et al. Correlation of Chest CT and RT-PCR Testing in Coronavirus Disease 2019 (COVID-19) in China: A Report of 1014 Cases. Radiology. 2020;26:200642.

[9] Shi H, Han X, Jiang N, et al. Radiological findings from 81 patients with COVID-19 pneumonia in Wuhan, China: a descriptive study. Lancet Infect Dis. 2020;20:425-434.

[10] Chen N, Zhou M, Dong X, et al. Epidemiological and clinical characteristics of 99 cases of 2019 novel coronavirus pneumonia in Wuhan, China: a descriptive study. Lancet. 2020;395:507-13.

[11] Huang C, Wang Y, Li X, et al. Clinical features of patients infected with 2019 novel coronavirus in Wuhan, China. Lancet. 2020;395:497506.

[12] Karcioğlu Ö. What is Coronaviruses, and How Can We Protect Ourselves? Phnx Med J. 2020;2:66-71.

[13] Xu X, Yu C, Qu J, et al. Imaging and clinical features of patients with 2019 novel coronavirus SARS-CoV-2. Eur J Nucl Med Mol Imaging. 2020;47:1275-1280.

[14] Cheng Z, Qin L, Cao Q, et al. Quantitative computed tomography of the coronavirus disease 2019 (COVID-19) pneumonia. Radiology of Infectious Diseases. 2020.

[15] Yang Y, Yang M, Shen C, et al. Evaluating the accuracy of different respiratory specimens in the laboratory diagnosis and monitoring the viral shedding ofn2019-nCoV infections. 2020.

[16] Pan F, Ye T, Sun P, et al. Time Course of Lung Changes On Chest CT During Recovery From 2019 Novel Coronavirus (COVID-19) Pneumonia. Radiology. 2020.

[17] Lei J, Li J, Li X, et al. CT Imaging of the 2019 Novel Coronavirus (2019-nCoV) Pneumonia. Radiology. 2020;295:18.

[18] Bernheim A, Mei X, Huang M, et al. Chest CT findings in coronavirus disease-19 (COVID-19): relationship to duration of infection. Radiology. 2020.

[19] Zhao W, Zhong Z, Xie X, et al. Relation Between Chest CT Findings and Clinical Conditions of Coronavirus Disease (COVID19) Pneumonia: A Multicenter Study. AJR Am J Roentgenol. 2020;214:1072-1077.

[20] Pan Y, Guan H, Zhou S, et al. Initial CT findings and temporal changes in patients with the novel coronavirus pneumonia (2019nCoV): a study of 63 patients in Wuhan, China. Eur Radiol. 2020.

[21] Li K, Wu J, Wu F, et al. The Clinical and Chest CT Features Associated With Severe and Critical COVID-19 Pneumonia. Invest Radiol. 2020;55:327-331.

How to cite this article: AfsinIpekci, Yonca Senem Akdeniz, Onur Tutar, Sabri Sirolu, Osman Simsek, Seda Özkan. The Clinical and Computed Tomography Findings of Patients with COVID-19. Signa Vitae. 2020;16(1):173-178. doi:10.22514/sv.2020.16.0023. 